\title{
Una adecuada actividad física en ancianos reduce la mortalidad independientemente de la presencia de enfermedad cardiovascular
}

Changes in physical activity, mortality, and incidence of coronary heart disease in older men

Wannamethee GS, Shaper AG y Walker M. Lancet 1998; 351: 1603-08

\section{Objetivo}

Evaluar la influencia de la actividad física regular y los cambios en la actividad física con respecto a la mortalidad global, la mortalidad cardiovascular y la incidencia de eventos coronarios en hombres mayores de 65 años.

\section{Diseño}

Estudio poblacional de cohortes.

\section{Lugar}

Veinticuatro ciudades en el Reino Unido.

\section{Participantes}

Se incluyeron 5934 hombres que pudieron ser contactados (91\%) de una cohorte de 7735 hombres previamente evaluados en 1978-80 por medio de una encuesta de salud que incluía el tipo e intensidad de la actividad física que realizaban. En 1992 los 5934 dieron nuevamente información acerca de su actividad física y fueron seguidos por 4 años más.

\section{Evaluación de los factores de riesgo}

Se evaluó: ocupación, tabaquismo, alcohol, peso, altura, historia médica en general y en particular de enfermedad coronaria, infarto agudo de miocardio (IAM), accidente cerebrovascular (ACV), medicaciones y percepción de su estado de salud. Los participantes fueron agrupados en seis categorías según el tipo, frecuencia e intensidad de la actividad física: inactiva, ocasional, leve, moderada, moderadamente vigorosa y vigorosa. Para recabar los datos se utilizaron encuestas previamente validadas y las mismas preguntas que en la primera evaluación (1978-80). El seguimiento fue de 3 años para los eventos coronarios y de 4 años para la mortalidad global.

\section{Resultados principales}

Estos se expresan en riesgo relativo ajustado. Las variables que se incluyeron en el ajuste fueron: edad, tabaquismo, índice de masa muscular, clase social, autopercepción del estado de salud y otros tipos de actividad física. Mortalidad Global:

Entre los 4311 pacientes sin enfermedad coronaria la mortalidad global fue más alta en los inactivos y decreció de una manera inversa con

Fuente de financiamiento: UK Department of Health. respecto a la actividad física, pero sin un beneficio adicional más allá de la actividad moderada. Debido al pequeño número de pacientes inactivos y vigorosos, se unieron a las dos categorías inmediatas adyacentes quedando cuatro grupos.

Tabla 1

\begin{tabular}{llll}
\hline Tipo de Actividad & N & Muertes & RR ajustado( IC 95\%) \\
\hline Inactivo/0casional & 1321 & $101(18,5 / 1000)$ & 1,00 \\
\hline Leve & 1011 & $48(11,4 / 1000)$ & $0,61(0,43-0,86)$ \\
\hline Moderada & 747 & $23(7.3 / 1000)$ & $0.50(0.31-0.79)$ \\
\hline Mod vigorosa-Vigorosa 1232 & $47(9,1 / 1000)$ & $0,65(0,45-0,94)$ \\
\hline
\end{tabular}

Cambios en actividad física y mortalidad global, cardiovasculary no cardiovascular:

Se agruparon en cuatro grupos en función de los cambios de actividad física: A: siempre inactivo; B: disminuyo actividad; C: incremento actividad y D: siempre activo (ver tabla 2). Los que incrementaron su actividad tuvieron menor mortalidád global, así como menor mortalidad cardiovascular y no cardiovascular.

Tabla 2

\begin{tabular}{|c|c|c|c|}
\hline Grupo $\mathrm{N}$ & $\begin{array}{l}\text { Mort. Global(\%o) } \\
\text { RRajustado }\end{array}$ & $\begin{array}{l}\text { M.Cardiovasc. }(\% o) \\
\text { RR ajustado }\end{array}$ & $\begin{array}{l}\text { M. NoCandiovasc. }(\% \circ) \\
\text { RR ajustado }\end{array}$ \\
\hline Grupo A 687 & $62(22 \% 0) 1.00$ & $25(8.8 \% 0) \quad 1.00$ & $37(13.1 \% 0) \quad 1.00$ \\
\hline \multirow[t]{2}{*}{ Grupo B 617} & $38(15 \%)$ & $21(8.2 \% 0)$ & $17(6.7 \% 0)$ \\
\hline & $0.75(0.50-1.14)$ & $1.02(0.56-1,86)$ & $0.56(0.31-1.02)$ \\
\hline \multirow[t]{2}{*}{ Grupo C 796} & $36(10 \%)$ & $17(5.1 \% 0)$ & $19(5.7 \% 0)$ \\
\hline & $0.55(0.36-0.84)$ & $0.66(0.35-1.23)$ & $0.48(0.27-0.85)$ \\
\hline \multirow[t]{2}{*}{ Grupo D 2160} & $81(9 \% 0)$ & $29(3.3 \% 0)$ & $52(5.7 \% 0)$ \\
\hline & $0.58(0.41-0.82)$ & $0.54(0.31-0.94)$ & $0.60(0.39-0.94)$ \\
\hline
\end{tabular}

La incidencia de eventos coronarios mostró un patrón similar de reducción de eventos en función de la actividad física o de su incremento.

\section{Conclusiones}

El mantenimiento y/o el cambio hacia una actividad física de regular a moderada reduce la mortalidad global y la incidencia de eventos coronarios mayores (fatales y no fatales) en ancianos, independientemente de poseêr o no enfermedad coronaria previamente detectada.

\section{COMENTARIO}

Para los médicos que atendemos ancianos este es un estudio trascendente y fortalece el concepto acerca del beneficio del ejercicio en los gerontes. Este estudio prospectivo demuestra que la actividad física moderada reduce la mortalidad global y todos los eventos coronarios independientemente de la actividad física previa del sujeto.

Además de la mejoría en eventos coronarios y mortalidad, existen cada vez más datos con respecto a otros beneficios de la actividad física en gerontes (principalmente en mayores de 75 años) tales como el mejoramiento en la tonicidad muscular, el equilibrio, la disminución del riesgo de caídas y de osteoporosis. El beneficio secundario de "sentirse mejor" que reportan nuestros viejos por la actividad física es no menos importante puesto que incide directamente en la calidad de vida. El trabajo demuestra que la actividad física regular y no necesariamente la vigorosa o deportiva, es la que reporta mayores beneficios. El se- gundo dato interesante consiste en que el incremento en la actividad física en los ancianos sedentarios, también es beneficioso.

Otros reportes que coinciden con el presente estudio, demuestran que actividades de fácil realización tales como caminatas contribuyen a aumentar la sobrevida ${ }^{1-3}$. Todavía no está claro cuáles son los mecanismos biológicos mediados para este efecto del ejercicio, pero no parece ser mediado sólo por los cambios de la presión arterial o del perfil lipídico? Las recomendaciones de ejercicio moderado, tales como las caminatas o las actividades recreacionales aeróbicas, son de baja tecnología, de potencialmente fácil aplicabilidad y de alto impacto $4-5$.

Es hora de que los médicos de atención primaria indiquemos enfáticamente actividad física regular a nuestros ancianos, a pesar de que el cumplimiento de las indicaciones médicas en lo que respecta a cambio de hábitos de vida sean tan difíciles de llevar a cabo.

\section{Dr. Manuel Montero Odasso}

Unidad de Medicina Familiar y Preventiva. Hospital Italiano de Buenos Aires.

\section{Referencias}

1. Sherman SE, D'agostina RB, CobbJL, Kannel WB. Does exercise reduce mortality rates in the elderly? Experience from the Framingham Heart Study. Am Heart J 1994;128:965-72

2. Shaper AG, Wannamethe G, Weatheral R. Physical activity and ischeamic heart disease in middle-aged men. Br Heart J 1991; 66:384-394

3. Hakim AA, Petrovich H, Burchfield CM, y col. Effects of walking on mortality among non-smoking retired men. N EngJ Med 1998; 338:94-99.

4. Edwards KE, Larson EB. Benefits of exercise for older adults. Clin Geriatr Med 1992:1: 35-50.

5. Edwards KE, Larson EB, Wagner E. Factors associeted with regular exercise in an elderly population J Am Board Fam Pract 1992; 5: 467-474. 